\title{
Oscillatory behavior of second-order nonlinear neutral differential equations
}

Jun Liu ${ }^{1 *}, X i$ Liu ${ }^{2}$ and Yuanhong $Y u^{3}$

*Correspondence:

liujunxei@126.com

${ }^{1}$ Institute of Applied Mathematics,

Qujing Normal University, Qujing

P.R. China

Full list of author information is

available at the end of the article

\begin{abstract}
We shall consider a class of second-order nonlinear neutral differential equations. Some new oscillation criteria are established by using the Riccati transformation technique. One example is given to show the applicability of the main results.
\end{abstract}

MSC: $34 \mathrm{~K} 11$

Keywords: Oscillation; Neutral differential equation; Riccati transformation

\section{Introduction}

In this paper, we study the oscillation of a class of second-order nonlinear differential equations,

$$
\left(r(t)\left(z^{\prime}(t)\right)^{\alpha}\right)^{\prime}+f(t, x(\sigma(t)))=0, \quad t \geq t_{0}>0,
$$

where $z(t)=x(t)-p(t) x(\tau(t)), \alpha>0$, and $\alpha$ is the ratio of two odd integers. The following assumptions are satisfied:

$\left(H_{1}\right) r, p \in C\left(\left[t_{0}, \infty\right), R\right), r(t)>0,0 \leq p(t) \leq p_{0}<1$.

$\left(H_{2}\right) \quad \tau \in C\left(\left[t_{0}, \infty\right), R\right), \tau(t) \leq t, \lim _{t \rightarrow \infty} \tau(t)=\infty$.

$\left(H_{3}\right) \quad \sigma \in C^{1}\left(\left[t_{0}, \infty\right), R\right), \sigma(t) \leq t, \sigma^{\prime}(t)>0, \lim _{t \rightarrow \infty} \sigma(t)=\infty$.

$\left(H_{4}\right) f \in C(R, R), u f(t, u)>0$ for all $u \neq 0$, and there exists a function $q(t) \in C\left(\left[t_{0}, \infty\right]\right.$, $[0, \infty))$ such that $|f(t, u)| \geq q(t)\left|u^{\alpha}\right|$.

Second-order and third-order differential equations are widely used in population dynamics, physics, technology and other fields. Many scholars have studied the oscillation of second-order differential equations [1-10]. Similarly, many scholars have studied the oscillation of third-order differential equations [11-14]. On this basis, this paper studies the second-order neutral differential Eq. (1), Some new oscillation criteria are established by using the Riccati transformation technique.

\section{Lemmas}

In order to establish the oscillation criterion of Eq. (1), we will give three lemmas.

(c) The Author(s) 2020. This article is licensed under a Creative Commons Attribution 4.0 International License, which permits use, sharing, adaptation, distribution and reproduction in any medium or format, as long as you give appropriate credit to the original author(s) and the source, provide a link to the Creative Commons licence, and indicate if changes were made. The images or other third party material in this article are included in the article's Creative Commons licence, unless indicated otherwise in a credit line to the material. If material is not included in the article's Creative Commons licence and your intended use is not permitted by statutory regulation or exceeds the permitted use, you will need to obtain permission directly from the copyright holder. To view a copy of this licence, visit http://creativecommons.org/licenses/by/4.0/. 
Lemma 2.1 Assume that

$$
\int_{t_{0}}^{\infty} r^{-\frac{1}{\alpha}}(t) d t=\infty
$$

and $x(t)$ is an eventually positive solution of Eq. (1). Then $z(t)$ has the following two possible cases:

(i) $z(t)>0, z^{\prime}(t)>0,\left(r(t)\left(z^{\prime}(t)\right)^{\alpha}\right)^{\prime} \leq 0$;

(ii) $z(t)<0, z^{\prime}(t)>0,\left(r(t)\left(z^{\prime}(t)\right)^{\alpha}\right)^{\prime} \leq 0$.

Proof Since $x(t)$ is an eventually positive solution of (1), there exists a $t_{1} \geq t_{0}$ such that $x(t)>0$, for $t \geq t_{1}$. From (1), we have

$$
\left(r(t)\left(z^{\prime}(t)\right)^{\alpha}\right)^{\prime} \leq 0
$$

hence $r(t)\left(z^{\prime}(t)\right)^{\alpha}$ is decreasing function and of one sign, therefore $z^{\prime}(t)$ is also of one sign, that is, there exists a $t_{2} \geq t_{1}$ such that, for $t \geq t_{2}, z^{\prime}(t)>0$ or $z^{\prime}(t)<0$.

If $z^{\prime}(t)>0$, we have (i) or (ii). Now, we prove that $z^{\prime}(t)<0$ will not happen.

If $z^{\prime}(t)<0$, we have

$$
r(t)\left(-z^{\prime}(t)\right)^{\alpha} \geq r\left(t_{2}\right)\left(-z^{\prime}\left(t_{2}\right)\right)^{\alpha}=K \geq 0,
$$

where $K=r\left(t_{2}\right)\left(-z^{\prime}\left(t_{2}\right)\right)^{\alpha} \geq 0$, that is,

$$
z^{\prime}(t) \leq-k^{\frac{1}{\alpha}} r^{-\frac{1}{\alpha}}(t)
$$

Integrating this inequality from $t_{2}$ to $t$, we have

$$
z(t) \leq z\left(t_{2}\right)-k^{\frac{1}{\alpha}} \int_{t_{2}}^{t} r^{-\frac{1}{\alpha}}(s) d s
$$

by condition (2), $\lim _{t \rightarrow \infty} z(t)=-\infty$. We will consider the following two cases.

Case 1. If $x(t)$ is unbounded, then there exists a sequence $\left\{t_{m}\right\}$, such that $\lim _{m \rightarrow \infty} t_{m}=\infty$ and $\lim _{m \rightarrow \infty} x\left(t_{m}\right)=\infty$, here $x\left(t_{m}\right)=\max \left\{x(s): t_{0} \leq s \leq t_{m}\right\}$. Hence, we have

$$
\begin{aligned}
x\left(\tau\left(t_{m}\right)\right) & =\max \left\{x(s): t_{0} \leq s \leq \tau\left(t_{m}\right)\right\} \\
& \leq \max \left\{x(s): t_{0} \leq s \leq t_{m}\right\}=x\left(t_{m}\right) .
\end{aligned}
$$

We get

$$
z\left(t_{m}\right)=x\left(t_{m}\right)-p\left(t_{m}\right) x\left(\tau\left(t_{m}\right)\right) \geq\left[1-p\left(t_{m}\right)\right] x\left(t_{m}\right)>0 .
$$

This contradicts $\lim _{t \rightarrow \infty} z(t)=-\infty$.

Case 2. If $x(t)$ is bounded, then $z(t)$ is bounded, this contradicts $\lim _{t \rightarrow \infty} z(t)=-\infty$. Hence, $z(t)$ satisfies one of the cases (i) and (ii). 
Lemma 2.2 Assume that $x(t)$ is a positive solution of Eq. (1) and $z(t)$ satisfies case (i) of Lemma 2.1, then

$$
z(t) \geq R(t) r^{\frac{1}{\alpha}}(t) z^{\prime}(t), \quad\left(\frac{z(t)}{R(t)}\right)^{\prime} \leq 0,
$$

where $R(t)=\int_{T}^{t} r^{-\frac{1}{\alpha}}(s) d s, T \geq t_{0}$.

Proof For $t>T \geq t_{0}$, we have

$$
z(t)=z(T)+\int_{T}^{t} \frac{r^{\frac{1}{\alpha}}(s) z^{\prime}(s)}{r^{\frac{1}{\alpha}}(s)} d s \geq r^{\frac{1}{\alpha}}(t) z^{\prime}(t) \int_{T}^{t} r^{-\frac{1}{\alpha}}(s) d s=R(t) r^{\frac{1}{\alpha}}(t) z^{\prime}(t) .
$$

Thus, we conclude that

$$
\left(\frac{z(t)}{R(t)}\right)^{\prime}=\frac{z^{\prime}(t) R(t)-z(t) R^{\prime}(t)}{R^{2}(t)} \leq \frac{z^{\prime}(t) R(t)-R(t) r^{\frac{1}{\alpha}}(t) z^{\prime}(t) r^{-\frac{1}{\alpha}}(t)}{R^{2}(t)}=0 .
$$

Lemma 2.3 Assume that $x(t)$ is an eventually positive solution of (1) and

$$
\limsup _{t \rightarrow \infty} \int_{\tau^{-1}(\sigma(t))}^{t}\left(\frac{1}{r(s)} \int_{s}^{t} q(u) d u\right)^{\frac{1}{\alpha}} d s>p_{0} .
$$

Then the impossibility for $z(t)$ satisfies case (ii) of Lemma 2.1.

Proof Assume that $z(t)$ satisfies case (ii) of Lemma 2.1, we have

$$
-z(t)=-x(t)+p(t) x(\tau(t))<p(t) x(\tau(t)) \leq p_{0} x(\tau(t)) .
$$

That is,

$$
x(\tau(t)) \geq-\frac{1}{p_{0}} z(t) .
$$

We deduce that

$$
x(t) \geq-\frac{1}{p_{0}} z\left(\tau^{-1}(t)\right), \quad x(\sigma(t)) \geq-\frac{1}{p_{0}} z\left(\tau^{-1}(\sigma(t))\right) .
$$

From $(1)$ and $\left(H_{4}\right)$, we have

$$
\left(r(t)\left(z^{\prime}(t)\right)^{\alpha}\right)^{\prime}+q(t)(x(\sigma(t)))^{\alpha} \leq 0 .
$$

We get

$$
\left(r(t)\left(z^{\prime}(t)\right)^{\alpha}\right)^{\prime}+q(t)\left(-\frac{1}{p_{0}}\right)^{\alpha} z^{\alpha}\left(\tau^{-1}(\sigma(t))\right) \leq 0 .
$$

Integrating this inequality from $s$ to $t$, we conclude that

$$
r(t)\left(z^{\prime}(t)\right)^{\alpha}-r(s)\left(z^{\prime}(s)\right)^{\alpha}-\frac{1}{p_{0}^{\alpha}} \int_{s}^{t} q(u) z^{\alpha}\left(\tau^{-1}(\sigma(u))\right) d u \leq 0 .
$$


That is,

$$
-z^{\prime}(s) \leq \frac{1}{p_{0}}\left(\frac{1}{r(s)} \int_{s}^{t} q(u) z^{\alpha}\left(\tau^{-1}(\sigma(u))\right) d u\right)^{\frac{1}{\alpha}} .
$$

Integrating this inequality from $\tau^{-1}(\sigma(t))$ to $t$, we get

$$
z\left(\tau^{-1}(\sigma(t))\right)-z(t) \leq \frac{1}{p_{0}} z\left(\tau^{-1}(\sigma(t))\right) \int_{\tau^{-1}(\sigma(t))}^{t}\left(\frac{1}{r(s)} \int_{s}^{t} q(u) d u\right)^{\frac{1}{\alpha}} d s
$$

Since $z(t)<0$, we have

$$
\int_{\tau^{-1}(\sigma(t))}^{t}\left(\frac{1}{r(s)} \int_{s}^{t} q(u) d u\right)^{\frac{1}{\alpha}} d s \leq p_{0}
$$

This contradicts (3). Thus the impossibility for $z(t)$ satisfies case (ii) of Lemma 2.1.

\section{Oscillation results}

Theorem 3.1 Assume that (2) and (3) be satisfied. If there exists a positive function $\rho \in$ $C^{1}\left(\left[t_{0}, \infty\right),(0, \infty)\right)$, such that, for all sufficiently large $T \geq t_{0}$,

$$
\int_{t_{0}}^{\infty}\left[\rho(t) \bar{Q}(t)-\frac{r(t)\left(\rho^{\prime}(t)\right)^{\alpha+1}}{(\alpha+1)^{\alpha+1} \rho^{\alpha}(t)}\right] d t=\infty
$$

where $\left.\bar{Q}(t)=Q(t) \frac{R^{\alpha}(\sigma(t))}{R^{\alpha}(t)}\right), Q(t)=q(t)[1+\bar{p}(\sigma(t))]^{\alpha}, \bar{p}(t)=p(t) \frac{R(\tau(t))}{R(t)}$, then Eq. (1) is oscillatory.

Proof Assume that $x(t)>0$. From Lemma 2.1, $z(t)$ satisfies one of the cases (i) and (ii). Case (i). Suppose that case (i) holds, from Lemma 2.2, we have

$$
\frac{z(t)}{R(t)} \leq \frac{z(\tau(t))}{R(\tau(t))}
$$

That is,

$$
z(\tau(t)) \geq R(\tau(t)) \frac{z(t)}{R(t)}
$$

We get

$$
z(t)=x(t)-p(t) x(\tau(t)) \leq x(t)-p(t) z(\tau(t)) \leq x(t)-p(t) R(\tau(t)) \frac{z(t)}{R(t)}
$$

That is,

$$
x(t) \geq\left[1+p(t) \frac{R(\tau(t))}{R(t)}\right] z(t)=[1+\bar{p}(t)] z(t),
$$

where $\bar{p}(t)=p(t) \frac{R(\tau(t))}{R(t)}$. 
From (1), we conclude that

$$
\left(r(t)\left(z^{\prime}(t)\right)^{\alpha}\right)^{\prime}+q(t) x^{\alpha}(\sigma(t)) \leq 0 .
$$

Then we have

$$
\left(r(t)\left(z^{\prime}(t)\right)^{\alpha}\right)^{\prime}+q(t)[1+\bar{p}(\sigma(t))]^{\alpha} z^{\alpha}(\sigma(t)) \leq 0
$$

That is,

$$
\left(r(t)\left(z^{\prime}(t)\right)^{\alpha}\right)^{\prime} \leq-Q(t) z^{\alpha}(\sigma(t))
$$

where $Q(t)=q(t)[1+\bar{p}(\sigma(t))]^{\alpha}$.

We define a function $w(t)$ of the generalized Riccati transformation by

$$
w(t)=\frac{\rho(t) r(t)\left(z^{\prime}(t)\right)^{\alpha}}{z^{\alpha}(t)}
$$

Then $w(t)>0$, from Lemma 2.2, we have $\frac{z(\sigma(t))}{R(\sigma(t))} \geq \frac{z(t)}{R(t)}$, that is, $\frac{z(\sigma(t))}{z(t)} \geq \frac{R(\sigma(t))}{R(t)}$.

Using the inequality [2]

$$
B u-A u^{\frac{\theta+1}{\theta}} \leq \frac{\theta^{\theta}}{(\theta+1)^{\theta+1}} \frac{B^{\theta+1}}{A^{\theta}}, \quad \theta>0, A>0, B \in R,
$$

we have

$$
\begin{aligned}
w^{\prime}(t) & =\rho^{\prime}(t) \frac{r(t)\left(z^{\prime}(t)\right)^{\alpha}}{z^{\alpha}(t)}+\rho(t) \frac{\left(r(t)\left(z^{\prime}(t)\right)^{\alpha}\right)^{\prime}}{z^{\alpha}(t)}-\rho(t) \frac{\alpha r(t)\left(z^{\prime}(t)\right)^{\alpha+1}}{z^{\alpha+1}(t)} \\
& \leq \frac{\rho^{\prime}(t)}{\rho(t)} w(t)-\rho(t) Q(t) \frac{z^{\alpha}(\sigma(t))}{z^{\alpha}(t)}-\frac{\alpha}{(\rho(t) r(t))^{1 / \alpha}} w^{\frac{\alpha+1}{\alpha}}(t) \\
& \leq \frac{\rho^{\prime}(t)}{\rho(t)} w(t)-\rho(t) Q(t) \frac{R^{\alpha}(\sigma(t))}{R^{\alpha}(t)}-\frac{\alpha}{(\rho(t) r(t))^{1 / \alpha}} w^{\frac{\alpha+1}{\alpha}}(t) \\
& \leq-\rho(t) \bar{Q}(t)+\frac{\rho^{\prime}(t)}{\rho(t)} w(t)-\frac{\alpha}{(\rho(t) r(t))^{1 / \alpha}} w^{\frac{\alpha+1}{\alpha}}(t) \\
& =-\rho(t) \bar{Q}(t)+\frac{r(t)\left(\rho^{\prime}(t)\right)^{\alpha+1}}{(\alpha+1)^{\alpha+1} \rho^{\alpha}(t)}
\end{aligned}
$$

where $\left.\bar{Q}(t)=Q(t) \frac{R^{\alpha}(\sigma(t))}{R^{\alpha}(t)}\right)$.

Integrating this inequality from $T$ to $t$, we have

$$
w(t) \leq w(T)-\int_{T}^{t}\left(\rho(s) \bar{Q}(s)-\frac{r(s)\left(\rho^{\prime}(s)\right)^{\alpha+1}}{(\alpha+1)^{\alpha+1} \rho^{\alpha}(s)}\right) d s
$$

From (4), we get $\lim w(t)_{t \rightarrow \infty}=-\infty$, this contradicts $w(t)>0$.

Case (ii). If $z(t)$ satisfies (ii), then due to Lemma 2.3, Eq. (1) is oscillatory. 
Theorem 3.2 Assume that (2) and (3) are satisfied. If there exists a positive function $\varphi \in$ $C^{1}\left(\left[t_{0}, \infty\right),(0, \infty)\right)$ such that, for all sufficiently large $T \geq t_{0}$,

$$
\int_{t_{0}}^{\infty}\left[\bar{Q}(t)-\frac{\varphi^{\alpha+1}(t)}{r^{1 / \alpha}(t)}\right] \exp \left[(\alpha+1) \int_{T}^{t} \frac{\varphi(s)}{r^{1 / \alpha}(s)} d s\right]=\infty
$$

then Eq. (1) is oscillatory.

Proof We use the counter-evidence method, suppose we have a non-oscillatory solution $x(t)$ of Eq. (1), as above, suppose that $x(t)$ is a positive solution of (1), by using Lemma 2.1, $z(t)$ satisfies one of (i) and (ii), we discuss each of the two cases separately.

Case (i). Assume that $z(t)$ has property (i), we obtain (5). We define a function $V(t)$ of a generalized Riccati transformation by

$$
V(t)=\frac{r(t)\left(z^{\prime}(t)\right)^{\alpha}}{z^{\alpha}(t)} .
$$

Then $V(t)>0$, using the Yang inequality $\frac{1}{p} a^{p}+\frac{1}{q} b^{q} \geq a b, \frac{1}{p}+\frac{1}{q}=1$, similar to (6), we have

$$
\begin{aligned}
V^{\prime}(t) & =\frac{\left(r(t)\left(z^{\prime}(t)\right)^{\alpha}\right)^{\prime}}{z^{\alpha}(t)}-\frac{\alpha r(t)\left(z^{\prime}(t)\right)^{\alpha+1}}{z^{\alpha+1}(t)} \\
& \leq-\bar{Q}(t)-\frac{\alpha}{r^{1 / \alpha}(t)} V^{\frac{\alpha+1}{\alpha}}(t) \\
& =-\left[\bar{Q}(t)-r^{-\frac{1}{\alpha}}(t) \varphi^{\alpha+1}(t)\right]-(\alpha+1) r^{-\frac{1}{\alpha}}(t)\left[\frac{1}{\alpha+1} \varphi^{\alpha+1}(t)+\frac{\alpha}{\alpha+1} V^{\frac{\alpha+1}{\alpha}}(t)\right] \\
& =-\left[\bar{Q}(t)-r^{-\frac{1}{\alpha}}(t) \varphi^{\alpha+1}(t)\right]-(\alpha+1) r^{-\frac{1}{\alpha}}(t) \varphi(t) V(t) .
\end{aligned}
$$

That is,

$$
V^{\prime}(t)+(\alpha+1) r^{-\frac{1}{\alpha}}(t) \varphi(t) V(t) \leq-\left[\bar{Q}(t)-r^{-\frac{1}{\alpha}}(t) \varphi^{\alpha+1}(t)\right] .
$$

We get

$$
\begin{gathered}
{\left[V^{\prime}(t)+(\alpha+1) r^{-\frac{1}{\alpha}}(t) \varphi(t) V(t)\right] \exp \left[(\alpha+1) \int_{T}^{t} \frac{\varphi(s)}{r^{1 / \alpha}(s)} d s\right.} \\
\leq-\left[\bar{Q}(t)-r^{-\frac{1}{\alpha}}(t) \varphi^{\alpha+1}(t)\right] \exp \left[(\alpha+1) \int_{T}^{t} \frac{\varphi(s)}{r^{1 / \alpha}(s)} d s .\right.
\end{gathered}
$$

That is,

$$
\begin{aligned}
& \left(V(t) \cdot \exp \left[(\alpha+1) \int_{T}^{t} r^{-\frac{1}{\alpha}}(s) \varphi(s) d s\right]\right)^{\prime} \\
& \quad \leq-\left[\bar{Q}(t)-r^{-\frac{1}{\alpha}}(t) \varphi^{\alpha+1}(t)\right] \exp \left[(\alpha+1) \int_{T}^{t} \frac{\varphi(s)}{r^{1 / \alpha}(s)} d s .\right.
\end{aligned}
$$

Integrating this inequality from $T$ to $t$, we get

$$
0 \leq V(t) \cdot \exp \left[(\alpha+1) \int_{T}^{t} r^{-\frac{1}{\alpha}}(s) \varphi(s) d s\right]
$$




$$
\leq V(T)-\int_{T}^{t}\left(\left[\bar{Q}(t)-r^{-\frac{1}{\alpha}}(t) \varphi^{\alpha+1}(t)\right] \exp \left[(\alpha+1) \int_{T}^{t} \frac{\varphi(s)}{r^{1 / \alpha}(s)} d s\right) d t\right.
$$

This contradicts (7).

Case (ii). If $z(t)$ satisfies (ii), then due to Lemma 2.3, Eq. (1) is oscillatory.

Example Consider the following equation:

$$
\left(\left(x(t)-p x(t-1)^{\prime}\right)^{\frac{1}{3}}\right)^{\prime}+q_{0} x^{\frac{1}{3}}(t-2)=0 .
$$

Comparing Eq. (8) with Eq. (1), let $r(t)=1, \alpha=\frac{1}{3}, \tau(t)=t-1, \sigma(t)=t-2, q(t)=q_{0}>0$, $p(t)=p<1$ is a positive constant. Choose $\rho(t)=t, \varphi(t)=1$, we now verify (3):

$$
\limsup _{t \rightarrow \infty} \int_{\tau^{-1}(\sigma(t))}^{t}\left(\frac{1}{r(s)} \int_{s}^{t} q(u) d u\right)^{1 / \alpha} d s=\limsup _{t \rightarrow \infty} \int_{t-1}^{t} q_{0}(t-s)^{3} d s=\frac{q_{0}}{4}>p_{0} .
$$

Therefore, if $\frac{q_{0}}{4}>p_{0}$, obviously, the conditions of Theorem 3.1 and Theorem 3.2 are satisfied, then Eq. (8) is oscillatory.

Then the conditions of Theorem 3.1 and Theorem 3.2 are satisfied.

\section{Acknowledgements}

The authors express their sincere gratitude to the editors and referees for careful reading of the manuscript and valuable suggestions, which helped to improve the paper. This research is supported by NNSF of P.R. China (Grant No. 11361048), NSF of Yunnan Province (Grant No. 2017FH001-014), and NSF of Qujing Normal University (Grant No. ZDKC2016002), P.R. China.

\section{Funding}

There is no source of funding for the study.

\section{Availability of data and materials}

Data sharing not applicable to this article as no datasets were generated or analyzed during the current study.

\section{Competing interests}

The authors declare that they have no competing interests.

\section{Authors' contributions}

All three authors contributed equally to this work. All authors read and approved the final manuscript.

\section{Authors' information}

${ }^{1}$ Institute of Applied Mathematics, Qujing Normal University, Qujing, Yunnan 655011, P.R. China, professor. ${ }^{2}$ School of Information Science and Engineering, Yunnan University, Kunming, Yunnan 650091, P.R. China, doctor. ${ }^{3}$ Academy of Mathematics and systems Science, China Academy Science, Beijing, 100190, P.R. China, researcher.

\section{Author details}

${ }^{1}$ Institute of Applied Mathematics, Qujing Normal University, Qujing, P.R. China. ${ }^{2}$ School of Information Science and Engineering, Yunnan University, Kunming, P.R. China. ${ }^{3}$ Academy of Mathematics and systems Science, China Academy Science, Beijing, P.R. China.

\section{Publisher's Note}

Springer Nature remains neutral with regard to jurisdictional claims in published maps and institutional affiliations.

Received: 18 February 2020 Accepted: 25 March 2020 Published online: 31 July 2020

\section{References}

1. Li, Q., Wang, R., Chen, F., Li, T.X.: Oscillation of second-order nonlinear delay differential equations with nonpositive neutral coefficients. Adv. Differ. Equ. 2015, 35 (2015)

2. Candan, T:: Oscillation behavior of second-order nonlinear neutral differential equations with distributed deviating arguments. Appl. Math. Comput. 262, 199-203 (2015)

3. Liu, J., Luo, H.Y., Liu, X.: Oscillation criteria for half-linear functional differential equation with damping. Therm. Sci. 18, $1537-1542(2014)$ 
4. Luo, H.Y., Liu, J., Liu, X.: Oscillation behavior of a class of new generalized Emden-Fowler equations. Therm. Sci. 18, 1567-1572 (2014)

5. Dzurina, J., Stavroulakis, L.P.: Oscillatory criteria for second order delay differential equations. Appl. Math. Comput. $140,445-453(2003)$

6. Li, W.T.: Interval oscillation of second-order half-linear functional differential equations. Appl. Math. Comput. 155, 451-468 (2004)

7. Liu, H., Meng, H., Liu, P.: Oscillation and asymptotic analysis on a new generalized Emden-Fowler equation. Appl. Math. Comput. 219, 2739-2748 (2012)

8. Manojlovic, J., Shoukaku, Y., Tanigawa, T., Yoshida, N.: Oscillation criteria for second order differential equations with positive and negative coefficients. Appl. Math. Comput. 181, 853-863 (2006)

9. Weng, A., Sun, J.: Oscillation of second order delay differential equations. Appl. Math. Comput. 198, 930-935 (2008)

10. Thandapani, E., Muthulakshmi, V., Graef, J.R.: Oscillation criteria for second order nonlinear neutral delay differential equations with positive and negative coefficients. Int. J. Pure Appl. Math. 70, 261-274 (2011)

11. Jiang, C.M., Jiang, Y., Li, T.X.: Asymptotic behavior of third-order differential equations with nonpositive neutral coefficients and distributed deviating arguments. Adv. Differ. Equ. 2016, 105 (2016)

12. Zhang, Q.X., Gao, L., Yu, Y.H.: Oscillation criteria for third-order neutral differential equations with continuously distributed delay. Appl. Math. Lett. 25, 1514-1519 (2012)

13. Aktas, M.F., Cakmak, D., Tiryaki, A.: On the qualitative behaviors of solutions of third-order nonlinear functional differential equations. Appl. Math. Lett. 24, 1849-1855 (2011)

14. Candan, T:: Oscillation criteria and asymptotic properties of solutions of third-order nonlinear neutral differential equations. Math. Methods Appl. Sci. 38, 1379-1392 (2015)

\section{Submit your manuscript to a SpringerOpen ${ }^{\circ}$ journal and benefit from:}

- Convenient online submission

- Rigorous peer review

Open access: articles freely available online

- High visibility within the field

- Retaining the copyright to your article 